\title{
ПРОНИКНОВЕНИЕ ДИСКРЕТНОЙ ФАЗЫ СТРУИ С ТЯЖЕЛОИ ПРИМЕСЬЮ В ПОПЕРЕЧНЫЙ РАВНОМЕРНЫЙ ПОТОК
}

Для распределения порошкообразного материала в потоке газа в ряде устройств (плазмохимических реакторах, камерах сгорания и т. д.) используются схемы смешения, основанные на вдуве струи с тяжелой примесью в сносящий поток газа. Для расчета и анализа работы подобных устройств необходимо оценить распределение дискретной фазы в потоке. Следует отметить, что аналогичная задача имеет место и при изучении рассеяния тяжелой примеси из дымовых труб, с той существенной разницей, что характерный масштаб устройства, формирующего дымовой факел, отличается более чем на порядок.

Математическая модель должна достаточно корректно учитывать два фактора, а именно взаимодействие дискретной и непрерывной фаз в поперечной струе и условия формирования струи с тяжелой примесью. Оценка указанных факторов может быть сделана только на основании экспериментальных данных; в настоящей работе анализируются данные по проникновению дискретной фазы в поток.

Развитие однофазной струи, вдуваемой в однородный поток, определяется в координатах $x / D, y / D$ углом вдува $\alpha_{20}$, коэффициентом истечения $\mu$, отношением плотностей $\varrho_{2} / \varrho_{1}$ и скоростей $V_{20} / V_{1}$ струи и потока, а при умеренном отношении плотностей безразмерным комплексом $\varrho_{2} V_{20}^{2} / \varrho_{1} V_{1}^{2}\left[{ }^{1-3}\right]$. Естественно ожидать, что и в рассматриваемом случае развитие непрерывной фазы характеризуется аналогичными параметрами. Для описания проникновения дискретной фазы в поток указанную систему параметров необходимо дополнить за счет критериев подобия, определяющих дисперсное течение в трубе (отношением плотностей $\mathrm{Q}_{p} / \mathrm{Q}_{2}$ и размеров $d_{s} / D$, числом Рейнольдса $\mathrm{Re}=V_{20} D / v_{2}$, расходной массовой концентрацией $x$ ), и критериев, определяющих движение частиц в поле течения, формируемом при вдувании струи в поток $\left(\varrho p / \varrho_{1}, \mathrm{Re}_{s 0}=\left|V_{s 0}-V_{1}\right| d_{s} / v_{1}\right)$. Первая группа этих критериев с изменением условий формирования струи может трансформироваться. Установить влияние указанных параметров в широком диапазоне их изменения, достаточном для выявления эмпирических корреляционных зависимостей, на распределение дискретной фазы представляется невозможным и едва ли целесообразным. Поэтому основной целью при постановке настоящей работы было получение необходимых исходных данных для построения физически обоснованной математической модели.

Экспери мент альн ая аэродинамическую трубу с 
$300 \times 350$ мм и длиной 600 мм. Средняя по сечению скорость потока составляла 9,5 м/сек. Поперечно к потоку вдувалась струя воздуха с примесью порошков электрокорунда. Среднерасходная скорость воздуха $\bar{v}$ в устье струи составляла $10 ; 23,8$ и 45 м/сек; среднемассовый размер порошков $d_{s} 16,32$ и 70 мкм и плотность материала порошка $3,92 / \mathrm{cm}^{3}$. Струя формировалась в вертикальном участке трубы диаметром $D 7,8 ; 12,2 ; 15,6$ и 26 мм. Для исключения существенного влияния дискретной фазы на течение непрерывной фазы начальная расходная массовая концентрация $x$ поддерживалась на уровне 0,3 . Оптическими лазерными методами измерялись скорость $V_{s}$ и плотность распределения @s дискретной фазы. Подробнее методика измерений и экспериментальная установка описаны в [ $\left.{ }^{4}\right]$.

Некоторые результаты экспериментального иссл едо в ания. Измерялись профили распределения дискретной фазы в плоскости симметрии струи вдоль продольной (вблизи выходного отверстия) и вертикальной координат в фиксированных сечениях при $x=50,200,400$ и 600 мл. Эти профили позволили определить дисперсионную ось струи, под которой понимается линия, проходящая через центр отверстия и далее через максимальные значения $\varrho_{s}$ дискретной фазы в нормальных сечениях струи.

Для оценки рассеяния примеси в поперечном направлении измерялись также профили $\varrho_{s}$ на уровне дисперсионной оси вдоль поперечной координаты. Здесь и далее принята декартова система координат, начало которой совпадает с центром отверстия струи, ось $x$ направлена вдоль потока, а ось $y$ вертикально вверх по истечению струи.

Возможности установки позволяли изменять скорость вдува струи, размер частиц и диаметр отверстия в довольно широких пределах. На рис. 1 представлены данные, подобранные по одному варьируемому параметру. Как видно, все указанные параметры в существенной мере влияют на распределение дискретной фазы в потоке, но выявить их роль в чистом виде не удается из-за указанной выше взаимосвязанности условий формирования струи и распределения дискретной фазы.

Проникновение дискретной фазы в поток. Анализ данных показывает, что в потоке дискретная фаза сепарируется от непрерывной фазы поперечной струи, причем этот процесс тем эффективнее, чем крупнее частицы примеси (при прочих равных условиях). Таким образом, для достаточно крупных частиц существенное взаимодействие фаз имеет место до сечения разделения фаз, что приводит к некоторому, относительно небольшому, увеличению глубины проникновения частиц за счет транспортировки газовой фазой струи, с одной стороны, и одновременному изменению их скорости, с другой. Нетрудно видеть, что указанные эффекты пропорциональны расстоянию взаимодействия или глубине проникновения газовой фазы. Следовательно, дисперсионная ось, которая является наиболее вероятной траекторией из всех возможных траекторий частиц, для достаточно крупных частиц может быть определена как траектория частицы с характерным размером фракции и средним начальным количеством движения. Для достаточно мелких частиц их собственное относительное движение за счет начального импульса играет небольшую роль, сепарация частиц диокретной фазы от непрерывной несущественна и распределение частиц определяется переносом их непрерывной фазой. Отсюда следует, что в первом приближении глубина проникновения дискретной фазы в поток может быть представлена суммой глубины проникновения непрерывной фазы и глубины проникновения частицы, имеющей размер, равный среднемассовому размеру дискретной фазы 

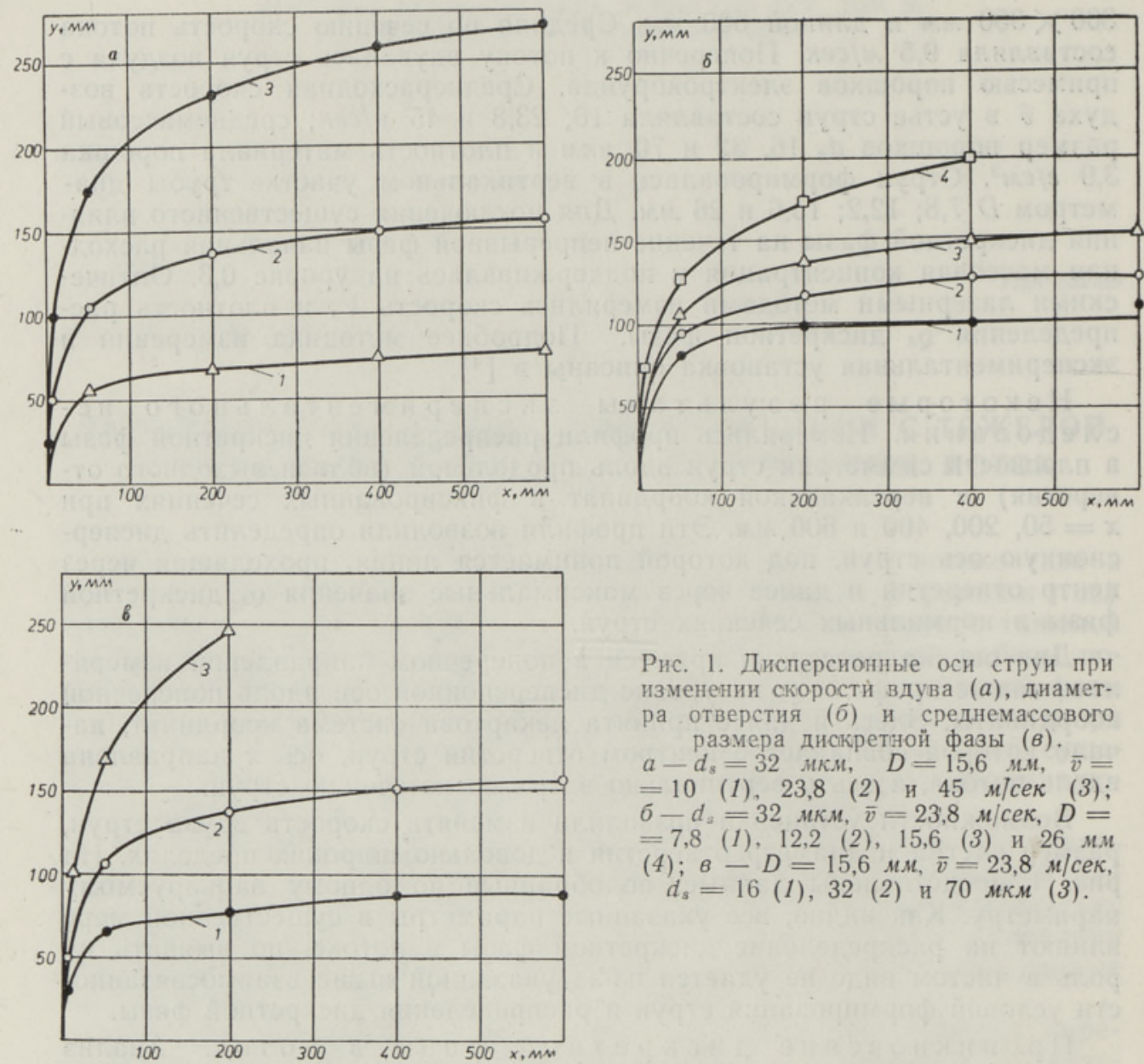

Рис. 1. Дисперсионные оси струи при изменении скоростй вдува $(a)$, диаметра отверстия (б) и среднемассового размера дискретной фазы $(8)$. $a-d_{s}=32$ мкм, $D=15,6$ м.,$\quad \bar{v}=$ $=10 \quad(1), \quad 23,8 \quad(2)$ и 45 м/сек (3); $\sigma-d_{2}=32$ мкм, $\bar{v}=23,8$ м/сек, $D=$ $=7,8 \quad(1), 12,2 \quad(2), 15,6 \quad(3)$ и 26 м.м (4); $в-D=15,6$ мм, $\bar{v}=23,8$ м/сек, $d_{s}=16(1), 32(2)$ и 70 мКм (3).

$$
y_{s}=\alpha y+\beta y_{p},
$$

где $y_{s}, y$ и $y_{p}$ - координаты возвышения дисперсионной оси, скоростной рси непрерывной фазы и частицы соответственно. Для практического использования зависимости типа (1) необходимо корректно оценить величины $y$ и $y_{p}$.

В случае пренебрежимо малого обратного влияния частиц на течение газа расчет $y$ вполне допустимо провести на основе данных исследования газовой поперечной струи $\left[{ }^{1-3}\right]$. Здесь для определения $y$ использована зависимость из $\left[{ }^{3}\right]$, которая получена в результате аналитического обобщения экспериментальных данных и поэтому может быть распространена на более сложные типы течения

$$
\begin{aligned}
& \frac{x_{*}}{D_{\mu}}=\frac{y_{*}}{D_{\mu}} \operatorname{ctg} \alpha_{20}+\frac{0,43}{q_{\mu}^{1,2}} \cdot\left(\frac{y_{*}}{D_{\mu}}\right)^{3}, \\
& \frac{y_{*}}{D_{\mu}}=\frac{y}{D_{\mu}}+0,59 q_{\mu}^{0,2},
\end{aligned}
$$

где $q_{\mu}=\varrho_{2} V_{2 \mu}^{2} / \varrho_{1} V_{1}^{2}-$ гидродинамический параметр, рассчитанный по условиям истечения струи, $x_{*}, y_{*}-$ декартовы координаты, отсчитывае- 
мые от полюса струи. В соответствии с экопериментальными данными, расчет возвышения оси струи по соотношению (2) производится до точки, где угол наклона касательной оси падает до значения $d y_{*} / d x *=$ $=\operatorname{tg} \alpha=0,087\left(\alpha=5^{\circ}\right)$. Далее избыточная скорость на оси струи становится сравнимой с интенсивностью пульсаций скорости, и струя теряет индивидуальность.

Для оценки величины $y_{p}$ в выражении (1) следует решить уравнения движения частицы. При этом необходимо иметь в виду, что число $\mathrm{Re}_{p}$ частицы по ходу ее движения меняется от $10^{2}-10^{3}$ до 0 , поэтому для получения аналитического решения существенным является выбор подходящей аппроксимации для стандартной кривой сопротивления. Здесь, как и в $\left.{ }^{5}\right]$, для коэффициента сопротивления частицы $c_{f}$ используется формула Клячко [ $\left.{ }^{6}\right]$, которая дает $2 \%$-ную корреляцию стандартной кривой сопротивления вплоть до чисел $\mathrm{Re}_{p}=400$ и правильный предельный переход при $\mathrm{Re}_{p} \rightarrow 0$ :

$$
c_{f}=\frac{24}{\operatorname{Re}_{p}}\left(1+\frac{1}{6} \operatorname{Re}_{p}^{2 / s}\right), \quad \operatorname{Re}_{p}=\frac{d_{p}\left|\hat{V}-V_{p}\right|}{v} .
$$

Из уравнений движения частицы

$$
\begin{aligned}
& \frac{d u_{p}^{\prime}}{d t}=c_{f} \frac{3 \varrho}{4 \varrho_{p} d_{p}}\left|\vec{V}-\vec{V}_{p}\right|\left(u-u_{p}\right), \\
& \frac{d v_{p}}{d t}=c_{f} \frac{3 \varrho}{4 \varrho_{p} d_{p}}\left|\vec{V}-\vec{V}_{p}\right|\left(v-v_{p}\right)
\end{aligned}
$$

при условии, что $u$ и $v=$ const (однородный поток), нетрудно получить соотношение между продольной и поперечной скоростями частицы

$$
\frac{u-u_{p}}{u-u_{p 0}}=\frac{v-v_{p}}{v-v_{p 0}}
$$

Важно отметить, что соотношение (5) не зависит от закона сопротивления частицы. Интегрирование системы уравнений (4) с использованием начальных условий $u_{p}(0)=u_{p 0}, v_{p}(0)=v_{p 0}$ и условия $v=0$ приводит к следующей системе параметрических уравнений для траектории частиц:

$$
\begin{gathered}
\frac{\alpha}{u-u_{p 0}} x_{p}=\frac{18}{\operatorname{Re}_{p 0}^{2 / 3}}\left(\bar{u}_{p}^{1 / 3}-1\right)+\frac{3}{2} \bar{u} \ln \frac{1+\frac{1}{6} \operatorname{Re}_{p 0}^{2 / 3} \bar{u}_{p}^{2 / 3}}{\left(1+\frac{1}{6} \operatorname{Re}_{p 0}^{2 / 3}\right) \bar{u}_{p}^{2 / 3}}+ \\
+\frac{18 \sqrt{6}}{\operatorname{Re}_{p 0}}\left[\operatorname{arctg}\left(\frac{\operatorname{Re}_{p 0}^{1 / 3}}{\sqrt{6}}\right)-\operatorname{arctg}\left(\frac{\bar{u}_{p}^{1 / 3} \operatorname{Re}_{p 0}^{1 / 3}}{\sqrt{6}}\right)\right],
\end{gathered}
$$

$\frac{\alpha}{v_{p 0}} y_{p}=\frac{18 \sqrt{6}}{\operatorname{Re}_{p 0}}\left[\frac{\operatorname{Re}_{p 0}^{1 / 3}}{\sqrt{6}}\left(1-\bar{v}_{p}^{1 / 3}\right)+\operatorname{arctg}\left(\frac{\bar{v}_{p}^{1 / 3} \operatorname{Re}_{p 0}^{1 / 3}}{\sqrt{6}}\right)-\operatorname{arctg}\left(\frac{\operatorname{Re}_{p 0}^{1 / 3}}{\sqrt{6}}\right)\right]$

где введены следующие обозначения

$$
\bar{u}_{p}=\frac{u-u_{p}}{u-u_{p 0}}, \quad \bar{v}_{p}=\frac{v_{p}}{v_{p 0}}, \quad \bar{u}=\frac{u}{u-u_{p 0}},
$$




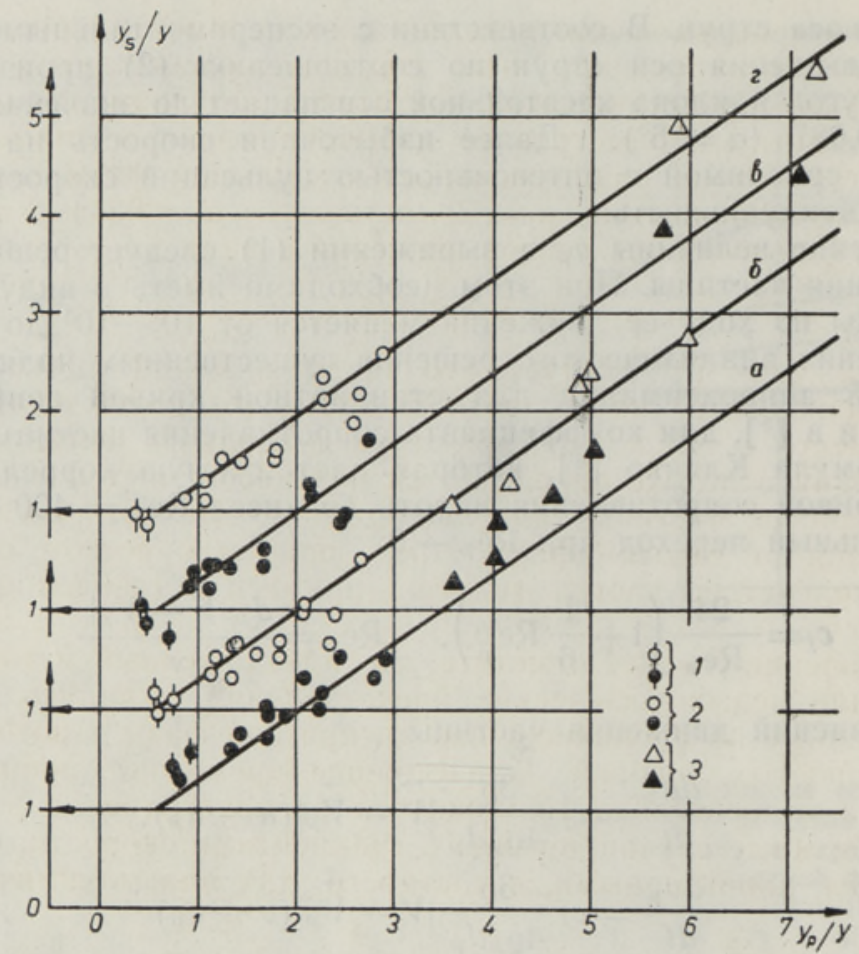

Рис. 2. Глубина проникновения тяжелой примеси в поток в сечениях $x=50(a), 200$ (б), 400 (в), 600 мм (2);

$$
1-d_{s}=16 ; 2-32,3-70 \text { мкм. }
$$

$$
\operatorname{Re}_{p 0}=\frac{\sqrt{v_{p 0}^{2}+\left(u-u_{p 0}\right)^{2}} d_{p}}{v}, \quad \alpha=\frac{18 \varrho v}{\varrho_{p} d_{p}^{2}},
$$

причем в соответствии с (5) $\bar{u}_{p}=\bar{v}_{p}$.

Из уравнений (6) нетрудно получить выражение для глубины проникновения частицы в поток, которую мы здесь определим как отсутствие относительного движения по вертикальной координате $\left(\bar{v}_{p}=0\right)$

$$
\frac{Y_{p}}{Y_{s t}}=\frac{18}{\operatorname{Re}_{p 0}^{2 / 3}}\left(1-\frac{\sqrt{6}}{\operatorname{Re}_{p 0}^{1 / 3}} \operatorname{arctg} \frac{\operatorname{Re}_{p 0}^{1 / 3}}{\sqrt{6}}\right), \quad Y_{s t}=\frac{v_{p 0}}{\alpha},
$$

где $Y_{s t}$ - глубина проникновения частицы в поток при стоксовом законе сопротивления. Из соотношений (7) непосредственно следует важный вывод о существенном влиянии закона сопротивления частицы на траекторию ее движения в потоке. Глубина проникновения частицы в поток определяется начальным числом $\mathrm{Re}_{p 0}$ частицы и характерным масштабом длины $Y_{s t}$ :

$$
Y_{p} / Y_{s t}=f\left(\operatorname{Re}_{p 0}\right) \text {. }
$$

Сопоставление с экспериментальны ми данны ми. В основу соотношения (1) была положена гипотеза о том, что коэффициенты $\alpha$ и $\beta$ постоянны. В какой мере это подтверждается экопериментом, можно видеть из рис. 2, где в координатах $y_{s} / y=f\left(y_{p} / y\right)$ пока- 
зана глубина проникновения тяжелой примеси в поток, определяемая по возвышению дисперсионной оси струи в соответствующем сечении. И хотя данные представлены в достаточно широком диапазоне изменения начальных параметров по скорости вдува и диаметру струи, группируются они по размеру частиц дискретной фазы. Значения постоянных, определенных из рис. 2 , составляют $\alpha=0,6$ и $\beta=0,7$. Соотношение (1) может быть теперь записано в виде

$$
y_{s}=0,6 y+0,7 y_{p} \text {. }
$$

Из выражения (9) следует, что частицы, для которых $y p / y \leqslant 0,57$ ( $y_{p}$ и $y$ вычисляются по уравнениям (2) и (6) соответственно), переносятся вместе с газовой фазой струи и, должно быть, характер их распределения в потоке определяется переносом частиц газовой фазой струи. Напротив, частицы тяжелой примеси, для которых $y_{p} / y \geqslant 0,57$, сепарируются от газовой фазы струи, причем с ростом соотношения $y_{p} / y$ взаимодействие частиц тяжелой примеси с газовой фазой струи уменьшается.

В заключение следует отметить, что предложенный метод позволяет на основании ясных физических представлений обобщить экопериментальные данные по проникновению дискретной фазы в поток для относительно простых условий. В настоящее время нет принципиальных возражений относительно возможности распространения этого метода на более сложные случаи течения с градиентами скорости, плотности и температуры, характерными, в частности, для плазмохимических реакторов.

\section{Л ИТ Е Р А Т У Р А}

1. Ив анов Ю. В., Эффективное сжигание надслойных порючих газов в топках паровых котлов, Таллин, 1959.

2. Шанд оров Т. С., Истечение в сносящий поток из отверстия в стенке канала и распространение струй в сносящем потоке, М., 1955.

3. З ло 6 ин В., Изв. АН ЭССР, Физ. Матем., 20, 66 (1971).

4. З лоби н В., Моос М., Изв. АН ЭССР, Физ. Матем., 25, 292 (1976).

5. R u d i n g e r, G., AIAA J., 12, No. 8, 1138 (1974).

6. Ф ук с Н. А., Механика аэрозолей, М., 1955.

Ннститут термофизики и электрофизики Академии наук Эстонской ССР
Поступила в редакцию $11 /$ XI 1976

\section{ZLOBIN, M. MOOS}

\section{HIIBSE JOA TAHKE FAASI ULATUVUS UHTLASES RISTVOOLUSES}

Esitatakse katseandmeid ühtlasesse ristvoolusesse puhutud hiibse joa dispersioontelgedest. Ristvooluse kiirus oli $9,5 \mathrm{~m} / \mathrm{s}$, joa diameeter $7,8-26 \mathrm{~mm}$, hiibse joa algkiirus $10-45 \mathrm{~m} / \mathrm{s}$ ja tolmutera keskmine diameeter $16-70 \mu \mathrm{m}$. Katseandmed üldistati järg. mise hüpoteesi abil: tahke faasi ulatuvus võrdub samasugust kiirust ning diameetrit omava üksiku tolmutera ulatuvuse ja joa gaasifaasi ulatuvuse lineaarse kombinatsiooniga. 
V. ZLOBIN, M. MOOS

\section{DISCRETE PHASE PENETRATION OF TWO-PHASE JET INTO A CONSTANT CROSS FLOW}

The results of experimental investigation on dispersion axis of two-phase jet are presented. Flow velocity was $9.5 \mathrm{~m} / \mathrm{s}$, tube diameter varied from 7.8 to $26 \mathrm{~mm}$, jet exit velocity from 10 to $45 \mathrm{~m} / \mathrm{s}$ and particles mean mass diameter from 16 to $70 \mu \mathrm{m}$.

The model presented for the calculation of dispersion axis is based on an assumption that discrete phase penetration is a linear combination of the penetration of particles having an initial velocity and size corresponding to the mean mass diameter of discrete phase and penetration of gaseous phase of jet. 